\title{
Echoes from Picasso: Explanation of an unusual artefact in optical coherence tomography
}

\author{
Miao Chu ${ }^{1,2}$, Gijs van Soest ${ }^{3}$, Miguel Ángel Martínez-Hervás-Alonso ${ }^{1}$, \\ Bernd Reisbeck ${ }^{1}$, Juan Luis Gutiérrez-Chico ${ }^{1}$ \\ ${ }^{1}$ Cardiology Department, Campo de Gibraltar Health Trust, Algeciras (Cádiz), Spain \\ ${ }^{2}$ Med-X Research Institute, School of Biomedical Engineering, \\ Shanghai Jiao Tong University, Shanghai, China \\ ${ }^{3}$ Erasmus Medisch Centrum, Rotterdam, Netherlands
}

This paper was guest edited by Dr. Evelyn Regar

\begin{abstract}
A patient referred for primary percutaneous coronary intervention presented with severe stenosis in the distal left main. After implanting a bifurcation-dedicated sirolimus-eluting stent (BIOSS-LIM $^{\mathrm{TM}}$, Balton, Warsaw, PL) angiography showed an apparently good angiographic result but with somewhat hazy appearance, requiring clarification by means of optical coherence tomography (OCT). OCT showed a region of severe underexpansion and a high ellipticity index (2.9) [1], corresponding with the hazy image (Fig. 1A). An interesting circular structure could be observed outside the vessel (Fig. 1A, arrow), mirroring the sheath of the optical catheter. This corresponds to an unusual expression of an artefact known as echo, happening when a strut is perpendicularly aligned with the OCT scan plane for a fairly large angle (Fig. 1A, asterisk). The light is then backscattered in the strut (Fig. 1B), and subsequently back to the catheter and to the opposite wall (Fig. 1B, dashed arrow), where it is reflected again, then back to the strut (Fig. 1C) and back to the catheter, where it is now detected (Fig. 1D), This creates echoes of
\end{abstract}

the backscattered structures throughout the beam pathway. The result is a mirror image where the optical catheter (normally absent from the OCT image; Fig. 1A, arrow) and the strut at the opposite wall (Fig. 1A, hash) can be recognised. The vicinity of structures, due to the severe underexpansion, favours the appearance of this artefact within the OCT field of view.

The curious image resembles a cubist artwork, like Picasso's portrait of Dora Maar (Fig. 1E), although attending to the physics underneath it would match better with Picasso's Girl before a Mirror.

\section{Conflict of interest: None declared}

\section{Reference}

1. Gutiérrez-Chico JL, Cortés C, Schincariol M, et al. Implantation of bioresorbable scaffolds under guidance of optical coherence tomography: Feasibility and pilot clinical results of a systematic protocol. Cardiol J. 2018; 25(4): 443-458, doi: 10.5603/ CJ.a2018.0055, indexed in Pubmed: 29774520.

Address for correspondence: Prof. Juan Luis Gutiérrez-Chico, MD, PhD, FESC, FACC, Head of the Cardiology Department, Hospital Punta de Europa, Crtra. Getares s/n, 11207 - Algeciras (Cádiz), Spain, tel: +49 (0) 17630585019 ,

+34 615 319370, e-mail: juanluis.gutierrezchico@ictra.es

Received: 24.07.2019 Accepted: 1.01.2020 


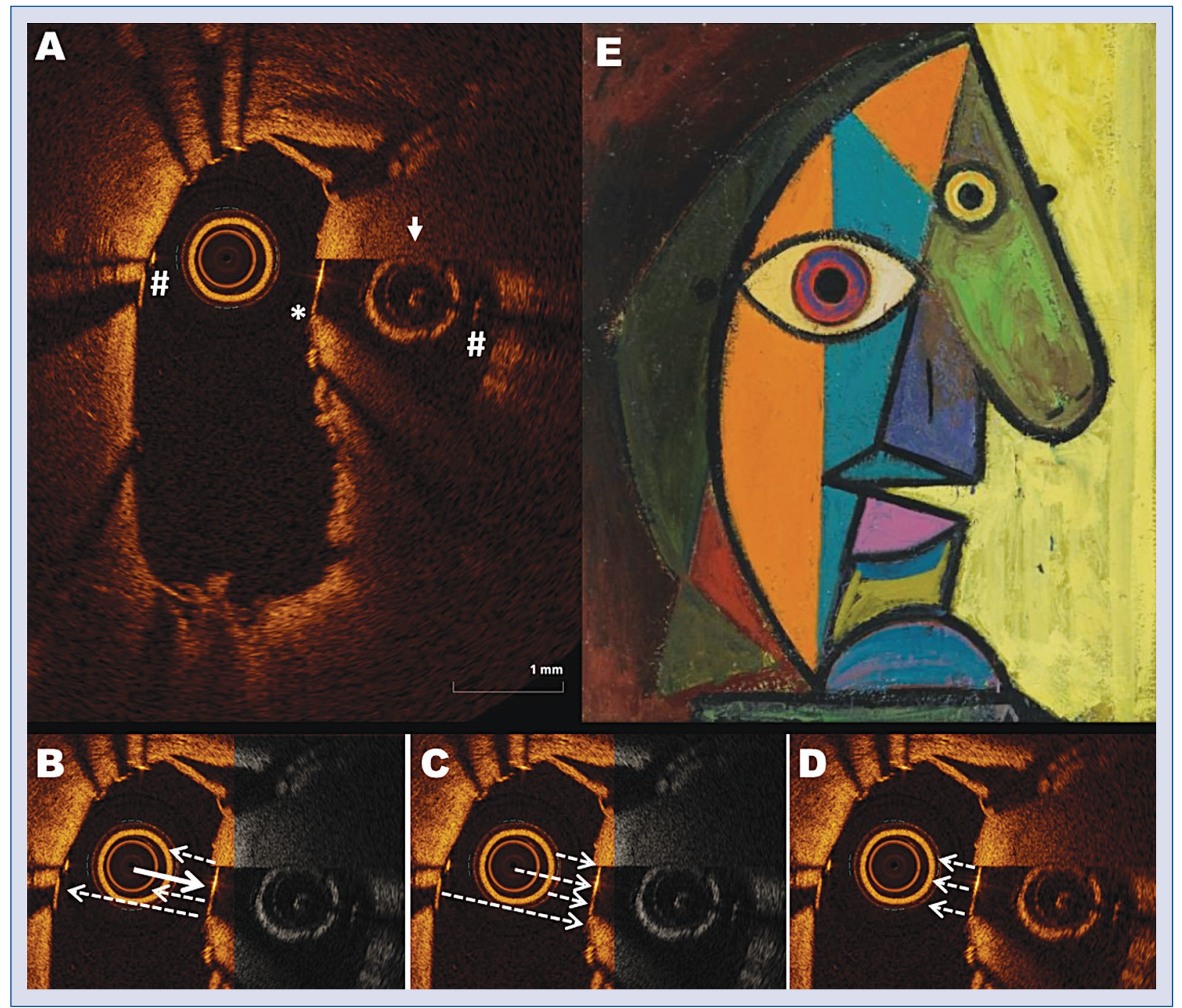

Figure 1. Marked echo artefact due to the vicinity of a large strut $\left({ }^{*}\right)$ to the optical catheter $(\#)$, depicting the optical catheter in the echo (arrow), usually absent from an optical coherence tomography image, and resembles an unusual cubist portrait. 